\title{
ANÁLISIS DEL USO DE AUDIOS DE MINDFULNESS EN LA ATENCIÓN Y LA CONCENTRACIÓN DE ESTUDIANTES DE FORMACIÓN PROFESIONAL
}

\section{ANALYSIS OF THE USE OF MINDFULNESS AUDIOS IN THE ATTENTION AND CONCENTRATION OF VOCATIONAL TRAINING STUDENTS}

\author{
José Carlos González Planells \\ pgplanells@gmail.com \\ Irene López Secanell \\ irlopez@florida-uni.es \\ Florida Universitària. Catarroja, Valencia (España)
}

Recibido: $13 / 05 / 2020$

Aceptado: 27/12/2020

\section{Resumen:}

El objetivo de la investigación fue analizar la influencia de los audios de Mindfulness sobre las capacidades disposicionales de una población adolescente para estar atentos y conscientes de la experiencia del momento presente. El ejercicio se implementó en un centro de Formación Profesional contando con 13 participantes y 18 estudiantes en el grupo de control. Se utilizaron como instrumentos de análisis los cuestionarios validados CAMM, EAP y MAAS-A. Se realizó un análisis de varianza que mostró que quienes recibieron la intervención presentaron pequeñas diferencias en las puntuaciones promedio de ítems concretos para los respectivos cuestionarios trabajados en el proyecto, no encontrándose resultados entre las puntuaciones globales de los cuestionarios entre clases.

Palabras Clave: mindfulness, educación profesional, adolescencia.

\begin{abstract}
The aim of the research was to analyze the impact of the use of exercise audios in mindfulness to verify an increase in the levels of full attention and academic concentration in an adolescent population. The exercise was implemented in a Vocational Training center, with 13 participants and 18 students in the control group. CAMM, EAP and MAAS-A validated questionnaires were used as analysis instruments. An analysis of variance was performed that showed that those who received the intervention presented small differences in the average scores of specific items for the respective questionnaires worked on in the project, with no results being found among the overall scores of the questionnaires between classes.
\end{abstract}

Keywords: mindfulness, professional education, adolescence. 


\section{Introducción}

El término Mindfulness es una traducción del término "Sati" (Smrti) proveniente de la lengua extinta pali, y que hace referencia al concepto de atención plena, consciencia plena o recuerdo (Siegel, Germer, y Olendzki, 2009). En los últimos años son diversas las investigaciones y programas que se han realizado entorno a esta técnica. En relación a las investigaciones, la mayoría de ellas se enfocan en dar evidencias sobre los distintos beneficios que aporta el uso continuado del mindfulness a nivel físico y psicológico (Anand y Sharma, 2014; Franco, Mañas, Cangas, y Gallego, 2011; Zoogman, Goldberg, Hoyt, y Miller, 2015; Wall, 2005; Broderick y Metz, 2009; Rosaen y Benn, 2006; Napoli, Krech y Holley, 2005; Biegel y Brown, 2010; Waters, Barsky, Ridd, y Allen, 2015). Conscientes de los beneficios, son numerosos los programas educativos que se han implementado en centros para ayudar al alumnado a autorregular sus emociones, su atención, y alcanzar un mayor bienestar psicológico. Un ejemplo de ello lo encontramos en los programas nacionales de mindfulness que se han introducido en centros educativos como los programas "Aulas felices", "TREVA", "Crecer Respirando" y "Escuelas Despiertas".

El mindfulness es una capacidad natural que posee el ser humano y por ello, es posible potenciarlo con entrenamiento, siendo la meditación la vía más utilizada para alcanzar un grado de atención plena elevado (Siegel, 2011; Lutz, Slagter, Dunne, y Davidson, 2008). Por este motivo, nace la necesidad de integrar diferentes técnicas basadas en la atención plena dentro del sistema educativo para reducir, en la medida de lo posible, los problemas exhibidos de aprendizaje, comportamiento, atención y problema de salud que sufren hoy en día los estudiantes durante la infancia y la adolescencia.

Aunque la presencia de investigaciones y programas de mindfulness es evidente, se observan dos limitaciones. Una de ellas es la falta de estudios con alumnado de formación profesional, ya que la mayoría se centran en alumnos de primaria, secundaria, universitaria o con personas que practican la meditación de forma externa a los centros educativos. La segunda, aunque los audios forman parte de las herramientas útiles para practicar el mindfulness (Almansa, et al. 2014), no se han encontrado trabajos realizados sobre el empleo de audios como técnica para mejorar la atención y la consciencia en estudiantes adolescentes en centros de Formación Profesional.

Por todo lo citado anteriormente, el presente trabajo tiene la finalidad de analizar la influencia de los audios de Mindfulness sobre las capacidades disposicionales de estudiantes de Grado Medio y Grado Superior de un centro de Valencia para estar atentos y conscientes de la experiencia del momento presente. Se pretende introducir al estudiante a este tipo de práctica para intentar lograr un aumento de su concentración y atención plena.

\section{Antecedentes teóricos}

\subsection{Concepto de la atención plena y su relación en el rendimiento académico}

La atención y el rendimiento académico es uno de los elementos claves en la etapa de la Formación Profesional. La atención es considerada como un factor clave para el proceso de manipulación y entendimiento de la información, que influye sobre la selección de ésta y establece prioridad en el procesamiento. Los estudiantes con déficits de atención, inquietos o distraídos, presentan dificultades en la compresión de la información que influye en el rendimiento académico, obteniendo calificaciones más bajas. Por el contrario, aquellos 
estudiantes que presentan mejores notas y cometen menos errores, son aquellos que demuestran tener una mejor atención selectiva y dividida (León, 2008; Napoli, et al., 2005).

La atención está estrechamente relacionada con el rendimiento académico. Según León (2008) el rendimiento académico es una especie de productividad del individuo, tratándose del producto final de la aplicación de su esfuerzo, matizado por sus actividades, rasgos y las percepciones de los cometidos asignados. Por otro lado, Carroll (1993), considera que las variables cognitivas (como la inteligencia, las aptitudes y conocimientos previos), las variables conativas y las variables afectivas (como la motivación y la personalidad), resultan ser los factores responsables y trascendentes del rendimiento académico.

\subsection{Beneficios del mindfulness en educación}

En el contexto educativo, el mindfulness o atención plena puede ser entendida como una base y una condición previa para la educación, ya que el mindfulness aporta numerosos beneficios que se traducen en una mejora de nuestra salud. Entre los beneficios psicológicos que aporta el mindfulness encontramos la reducción de los niveles de estrés (Anand y Sharma, 2014), la reducción de los niveles de ansiedad (Franco, Mañas, Cangas y Gallego, 2011; Napoli, Krech y Holley, 2005), la reducción de los niveles de depresión (Zoogman, Goldberg, Hoyt y Miller, 2015), la reducción de los niveles de afecto negativo y incremento de los niveles de afecto positivo y reducción de los niveles de cansancio, dolor y quejas psicosomáticas (Broderick y Metz, 2009). También encontramos beneficios relacionados con el aumento de sentimientos y constructos positivos (Wall, 2005) y la mejora de los niveles de optimismo y del auto-concepto (Franco, et al., 2011); beneficios en relación a la regulación emocional como mayor conciencia y claridad emocional (Broderick y Metz, 2009) y autocontrol de emociones negativas (Broderick y Metz, 2009; Rosaen y Benn, 2006); beneficios en la función cognitiva como el aumento en los niveles de atención (Napoli, et al., 2005) y la autorregulación y función ejecutiva (Biegel y Brown, 2010); beneficios en el ámbito social y escolar (Biegel y Brown, 2010; Napoli, et al., 2005).

\section{Material y Métodos}

\subsection{Objetivos e hipótesis}

Esta investigación tiene el objetivo de analizar la influencia de los audios de Mindfulness sobre las capacidades disposicionales de estudiantes adolescentes para estar atentos y conscientes de la experiencia del momento presente. En base a este objetivo la hipótesis que se plantea en el presente trabajo es que la clase Mindfulness donde se emplearán los audios presentará una mejora en la atención y la concentración en comparación con el grupo control.

\subsection{Metodología}

Se empleó una metodología cuantitativa mediante los cuestionarios validados CAMM, EAP y MAAS-A, a partir de los cuales se obtuvieron los resultados numéricos y cuantificables que posteriormente fueron analizados estadísticamente. Para ello, se dispuso de dos clases, donde teníamos por un lado la clase denominada Clase Mindfulness, la cual realizó técnicas de audio de mindfulness de manera periódica, y otra clase denominada Clase Control, que no recibió ningún tipo de ejercitación en la meditación. En ambas clases se llevó a cabo la realización de los mismos cuestionarios. 


\subsection{Participantes}

La clase control pertenecía al Módulo de Electricidad y Automatismos Electrónicos de un centro integrado público de formación profesional Grado Medio y Grado Superior, perteneciente a la provincia de Valencia. En el Grupo Control eran 18 estudiantes, todos ellos varones, mientras que en el grupo Mindfulness eran 13, encontrándonos una mujer y doce varones. Por otro lado, el rango de edad queda comprendido entre los 16 y los 21 años.

\subsection{Instrumentos de análisis, medidas y variables estudiadas}

Los cuestionarios utilizados fueron los siguientes:

- MAAS-A (Mindful Attention Awareness Scale-Adolescent): consiste en un cuestionario compuesto por 14 ítems, medido con una escala tipo Likert de seis puntos (1= casi siempre y 6 = casi nunca), teniendo, por tanto, una puntuación mínima de 14 puntos y una puntuación máxima de 84 . Una puntuación elevada, representa un mayor grado en mindfulness, capacidad de atención y consciencia plena. La escala evalúa el rasgo de consciencia plena, midiendo la frecuencia de la atención abierta y receptiva de las situaciones y experiencias que tienen lugar. Respecto a la fiabilidad del cuestionario, autores como Calvete, Sampedro e Orue (2014), en el estudio de adaptación del MAASA, señalan que obtuvieron un alfa de Cronbach que oscilaba entre 0.82 y 0.84 , así como la presencia de un único factor que explicaba el $32.52 \%$ de la varianza en una muestra en adolescentes. Para nuestro caso en particular, se obtuvieron en la población donde recibió ejercitación en Mindfulness un alfa de Cronbach de 0.84 , mientras que en la clase Control de un 0.86 , resultando como promedio un valor de 0.85 .

- CAMM (Child and Adolescent Mindfulness Measure): se trata de una escala psicológica sencilla, que evalúa de forma global, la capacidad de un niño/adolescente de estar atentos y ser conscientes de la experiencia que están sintiendo en cada momento presente (Timoneda, 2017). En cuanto a la escala, es de tipo Likert, compuesta de cinco puntos (1 $=$ Nunca a $5=$ Siempre), donde puntuaciones altas se correlacionan con puntuaciones negativas (bajo nivel mindfulness) y puntuaciones bajas, se relacionan con puntuaciones positivas (alto nivel mindfulness). Cuenta con 10 preguntas, por lo que su puntuación máxima por test es de 50 y su puntuación mínima es de 10. En cuanto a la fiabilidad para el cuestionario CAMM, autores como Greco, Baer y Smith (2011) lo validaron utilizando correlaciones bivariadas y parciales con variables relevantes. Los resultados sugieren que el CAMM es una medida apropiada para el desarrollo con una consistencia interna adecuada. En nuestro caso en particular, se obtuvo unos coeficientes de alfa de 0.69 para la clase Mindfulness y de 0.80 para la clase control, siendo el promedio de ambas de 0.74.

- EAP (Escala de Atención Plena en el Ámbito Escolar): se trata de un instrumento compuesto por 12 ítems, presentada dentro de una escala tipo Likert, comprendida por cinco intervalos (que van desde $1=$ nunca hasta $5=$ Siempre), donde puntuaciones elevadas muestran una alta capacidad mindfulness. La consistencia interna del cuestionario (Alfa de Cronbach) y la estabilidad Test-Retest, es muy aceptable $(0,84)$. En cuanto a la puntuación máxima por test, es de 60 puntos y su puntuación mínima es de 12 puntos. La consistencia interna del cuestionario EAP indicada en León (2008), muestra un alfa de Cronbach de 0,84 y una estabilidad test-retest de 0,78. En nuestro estudio, para la clase Mindfulness se obtuvo un valor de alfa de 0.77 , mientras que para la clase fue de 0.81 , obteniendo como promedio de ambas un alfa de Cronbach de 0.79.

En cuanto a las variables estudiadas fueron: Hora y Día de la realización del Cuestionario, Clase y Tipo de Cuestionario. 


\subsection{Análisis de la información}

Para la elaboración de gráficos y manejo de datos se trabajó con programa Microsoft Excel y para el cálculo de estadística se utilizó el programa Statgraphics Centurion, dónde se calculó la comparación de las medidas de las variables estudiadas.

\subsection{Diseño y estructura de las sesiones}

Los audios que se han utilizado en este estudio se apoyan en el libro de Simón y Alonso (2013), denominado Meditaciones de Mindfulness y Autocompasión. El motivo por el que se escogió este libro de audio fue por las capacidades de aprender a profundizar en la prácticas mindfulness que ofrece. Los audios seleccionados fueron los siguientes: Audio I: Meditación Preparatoria (10:13 min.), Audio II: Meditación Preparatoria (10:13 min.), Audio III: Meditación del Anhelo de Felicidad (22:22 min), Audio IV: Meditación del Anhelo de Felicidad (22:22 min), Audio V: Meditación Centrante. (15:24 min.), Audio VI: Meditación de la Bondad Amorosa y la Autocompasión (21:56 min.).

En cuanto a la organización del estudio, los alumnos realizaron un total de 18 cuestionarios por estudiante, correspondiéndose a 402 cuestionarios realizados en total. Los cuestionarios se dividieron en dos partes: la primera parte denominada Inicio en la Meditación y la segunda parte denominada Meditación. En cada parte, los estudiantes hicieron un total de 3 tests por cuestionario, correspondiéndose a un total de 9 cuestionarios por parte y estudiante. Con ello, se compararon la puntuación de la primera parte con la puntuación de la segunda parte, para ver la mejora en la meditación entre las dos partes, así como entre las dos clases, Clase Control y Clase Mindfulness.

El inicio de las sesiones tuvo lugar el día 03/02/2020 y tuvo como fecha final el 03/04/2020, estructurándose de la siguiente forma:

- La duración de la primera parte fue de tres semanas (03/02/2020 hasta el 21/02/2020). En la primera semana se diseñó la parte Inicio en la Meditación, que consistió en no realizar ningún tipo de Audio en Meditación, sirviendo como cuestionario Pre-Test para conocer así el nivel inicial de los estudiantes de ambas clases. En las dos semanas posteriores, se inició la introducción de los audios todos ellos, enfocados a la introducción del tema. En cuanto a los cuestionarios, se concentraron los 9 cuestionarios/alumno de la primera parte en las tres semanas.

- La segunda parte tuvo lugar en 5 semanas (24/02/2020 hasta el 03/04/2020). En la segunda parte denominada Meditación, la realización de los cuestionarios se esparcieron en una duración mayor para acumular así el mayor número de horas en ejercicios de meditación, teniendo por tanto la realización de un test/persona durante tres semanas seguidas. Posteriormente, en las últimas dos semanas, se concentraron los restantes cuestionarios. En cuanto a los audios, éstos se practicaron durante todas las semanas hasta la semana 7 (incluida), dejando sin la ejercitación del audio únicamente la Semana 8 , que se correspondía con la última semana del proyecto.

A continuación, se muestra la distribución de las sesiones del proyecto: 

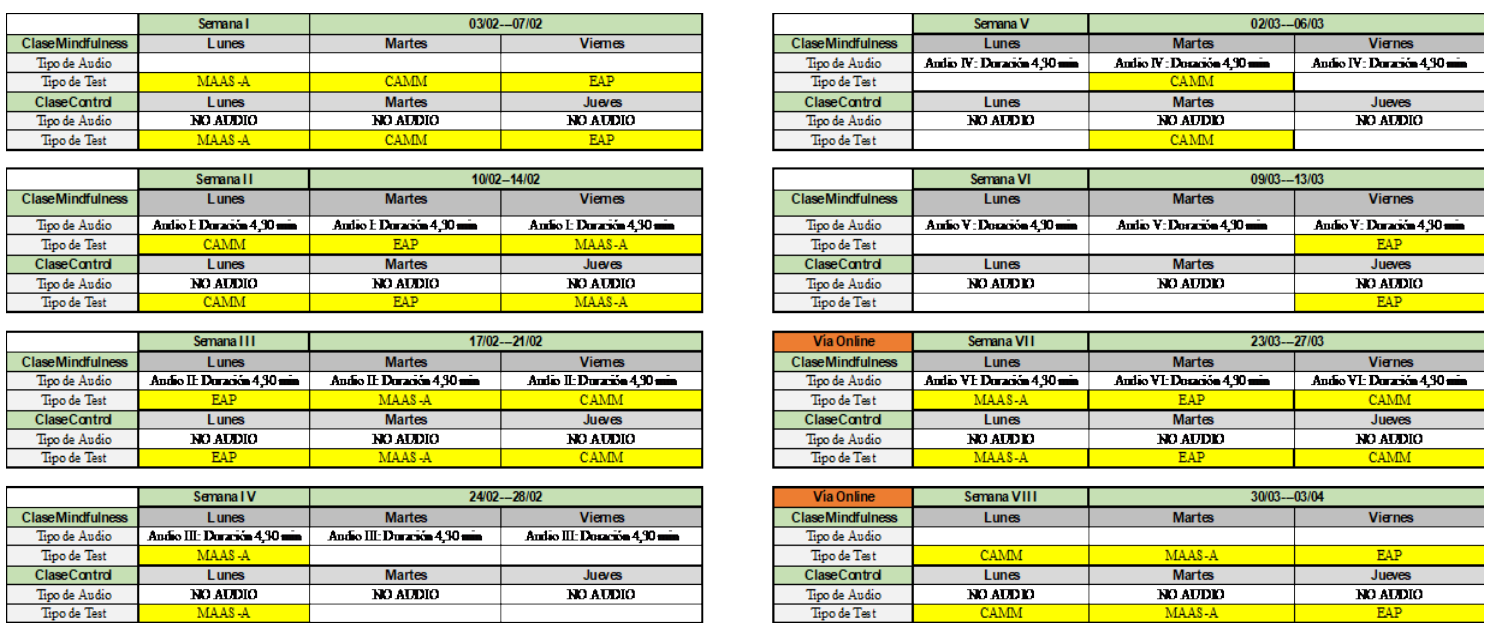

Figura 1. Distribución de las sesiones.

\section{Resultados}

\subsection{Estudio analítico del cuestionario MAAS-A para la Clase Mindfulness y la Clase Control}

\subsubsection{Análisis Global del Cuestionario MAAS-A}

Los resultados mostrados en el análisis de los datos para el cuestionario MAAS-A, muestran que, por lo general en ambas clases, no ha habido una variación significativa de respuesta entre sesiones. Los resultados muestran, que los alumnos han ido contestando de manera igualitaria y constante los cuestionarios, repitiéndose el mismo patrón de respuesta en cada sesión. A continuación, se muestra la evolución del promedio de respuesta de la Parte I (las tres primeras sesiones) con la Parte II (las tres sesiones restantes), tanto para la clase Control como para la Clase Mindfulness:

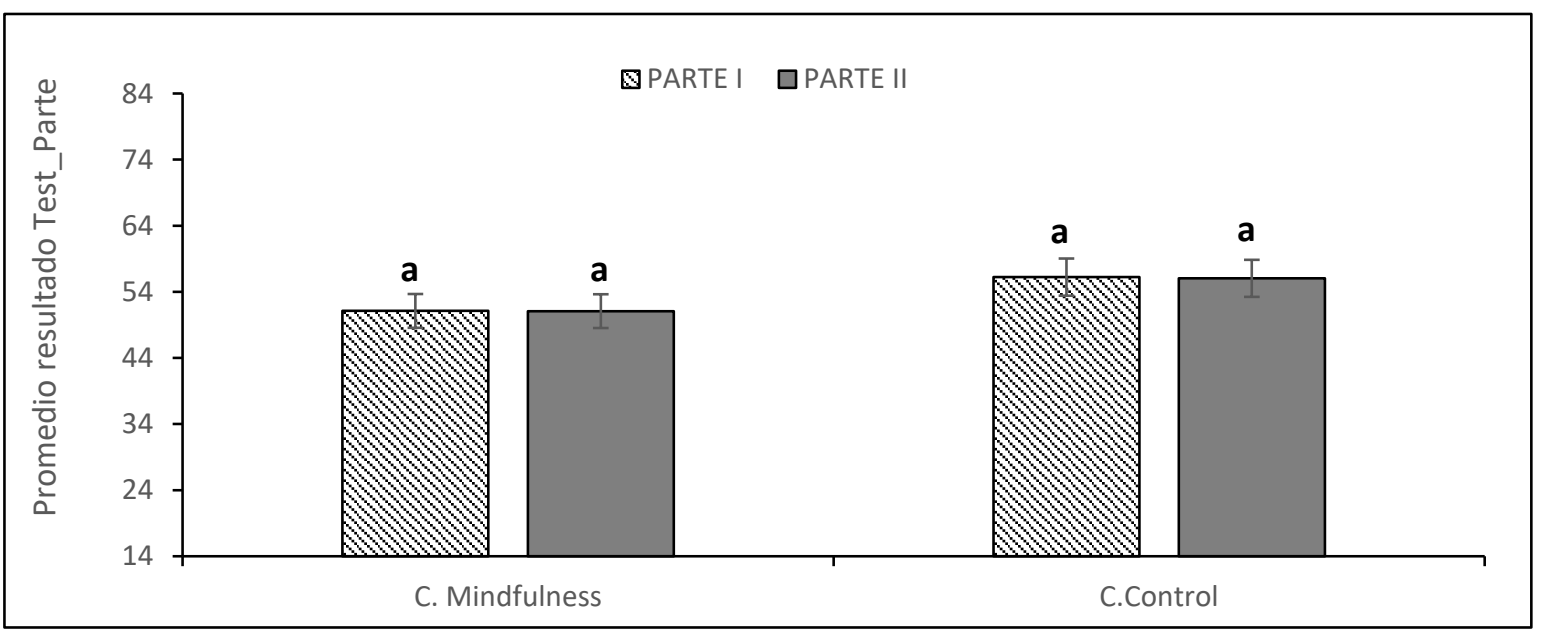

Figura 2. Evolución del promedio respuestas del Test MAAS-A de la Parte I y Parte II con el tipo clase de estudio. 
Se observa que no ha habido una diferencia significativa de mejoría entre la puntuación inicial (Parte I) y la puntuación final (Parte II), tanto para la clase Control, como para la clase Mindfulness. De igual modo, al compararse las puntuaciones promedio total entre ambas clases, se observa que la clase Control muestra una puntuación más elevada que la clase Mindfulness, siendo estas diferencias no significativas. Estos resultados coinciden con resultados realizados en otros estudios del MAAS-A, tal y como expresaron Bruin, Zijlstra, Weijer-Bergsma, y Bögels (2011), donde se mostró que los adolescentes sin experiencia en meditación obtuvieron puntuaciones más altas en atención plena que aquellos que recibieron experiencias en meditación. El valor de la puntuación, se observa un nivel medio-alto en Atención Plena en ambas clases.

En cuanto a la variable "Momento del día" y variable "Día" no han existido diferencias significativas en ambos casos (Véase en la Tabla 1).

Tabla 1.

Cuadro resumen entre las variables Momento del día y Día, para la clase Control como para la clase Mindfulness para el cuestionario MAAS-A.

\begin{tabular}{|c|c|c|c|c|c|}
\hline \multirow[b]{2}{*}{ Tipo de Clase } & \multirow[b]{2}{*}{ Momento del día } & \multicolumn{3}{|c|}{ Desviación } & \multirow[b]{2}{*}{ Valor-P } \\
\hline & & Promedio & Estándar & Razón-F & \\
\hline C. Mindfulness & Final de la mañana & 51 & 11,37 & 0,14 & 0,7061 \\
\hline C. Mindfulness & Primera hora & 50 & 12,15 & & \\
\hline C. Control & Final de la mañana & 56 & 12,80 & 0,07 & 0,9281 \\
\hline C. Control & Media mañana & 57 & 9,86 & & \\
\hline C. Control & Primera hora & 56 & 6,20 & & \\
\hline C. Mindfulness & Lunes & 51 & 11,56 & 0,07 & 0,9305 \\
\hline C. Mindfulness & Martes & 50 & 12,15 & & \\
\hline C. Mindfulness & Viernes & 51 & 11,31 & & \\
\hline C. Control & Jueves & 57 & 9,86 & 0,07 & 0,9281 \\
\hline C. Control & Lunes & 56 & 12,80 & & \\
\hline C. Control & Martes & 56 & 6,20 & & \\
\hline
\end{tabular}

Fuente: elaboración propia

\subsubsection{Análisis del Cuestionario MAAS-A estudiado por Ítem}

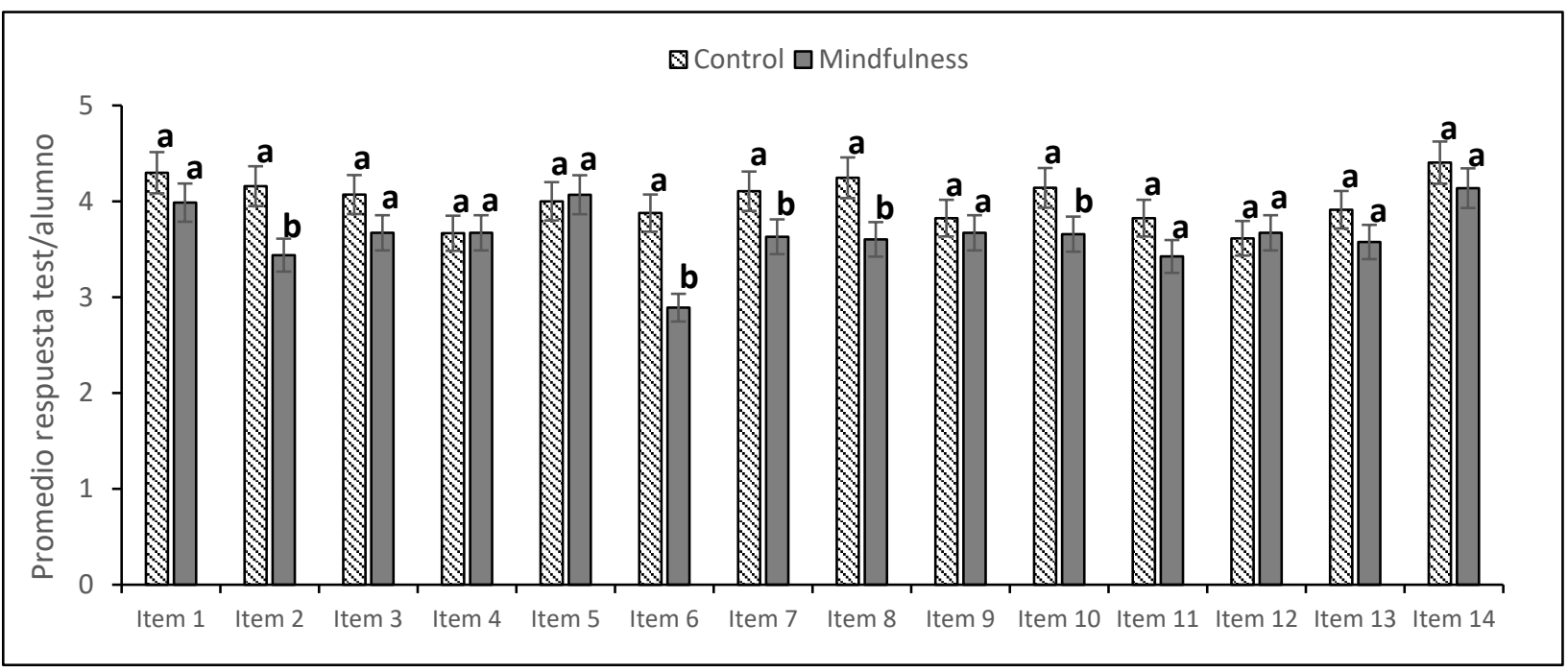

Figura 4. Comparación por Ítem y clase de estudio de los ítems comprendidos en el cuestionario MAAS-A. 


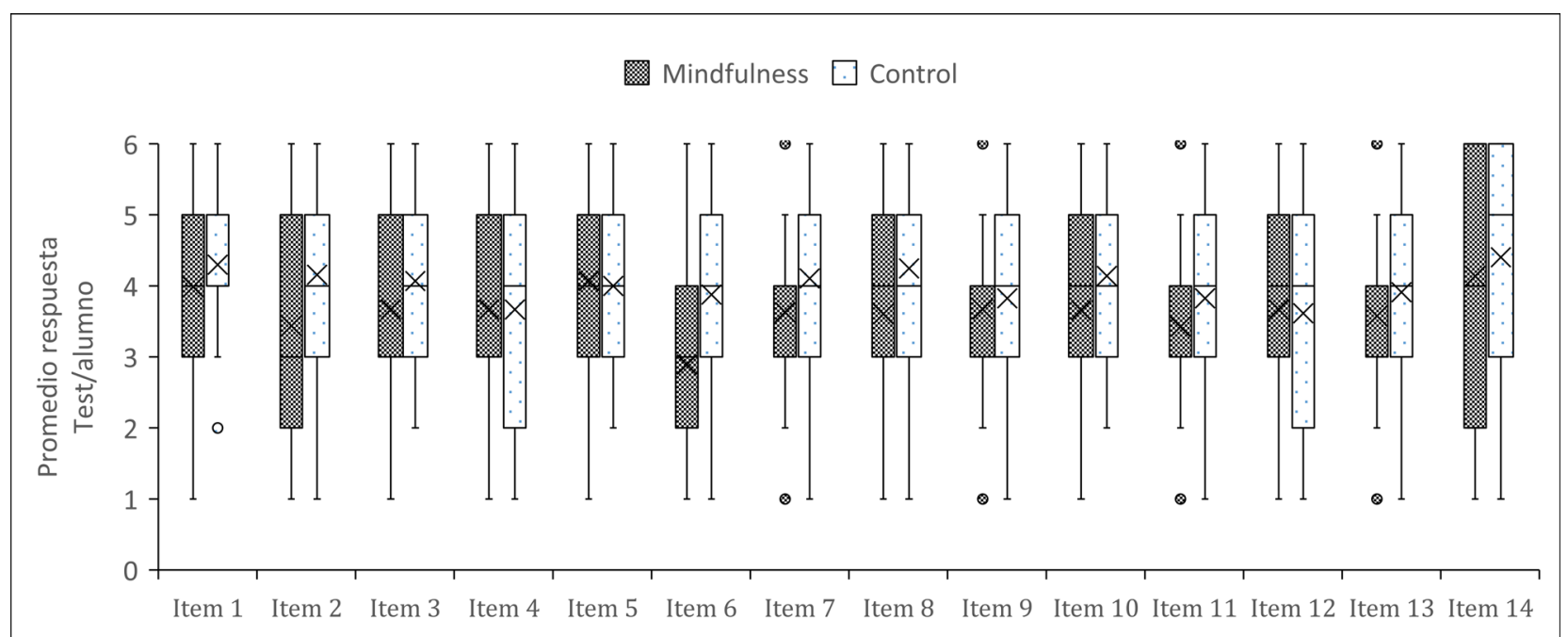

Figura 5. Comparación del rango de respuesta por alumno y clase para los respectivos ítems del cuestionario MAAS-A.

En el presente punto se analizó el promedio respuesta de cada ítem dentro de la calificación de la escala del cuestionario MAAS-A para ambas clases del estudio. Se observa que, por lo general, el promedio de respuesta de los distintos ítems pertenecientes a la escala MAAS es muy parejo en la mayoría de las cuestiones, presentando una ligera puntuación mayor en la clase Control que en la clase Mindfulness, contrastándose así con lo mencionado anteriormente por Bruin et al. (2011). En cuanto a diferencias observadas en las respuestas, se vio diferencias significativas entre las respuestas de ambas clases para los ítems 2 (Rompo o derramo cosas por descuido, por no prestar atención), 6 (Me olvido del nombre de una persona casi tan pronto como me lo dicen), 7 (Parece como si funcionara en piloto automático), 8 (Hago las actividades con prisa, sin estar realmente atento a ellas) y 10 (Hago trabajos o tareas de forma automática, sin darme cuenta de lo que estoy haciendo). En todas ellas, la clase Mindfulness mostró un valor inferior medio de respuesta/alumno respecto a la clase Control. Una posible hipótesis que se plantea es quizá que los alumnos de la clase Mindfulness, al explicarse el tipo de pregunta y entenderla, fueron más conscientes al contestar el Ítem, mostrando así un valor inferior que respecto a la clase Control, en la cual no hubo explicación de las preguntas y contestaron posiblemente con puntuaciones más elevadas en atención plena, sin ser correspondidas con la realidad. En el resto de los ítems, la igualdad del valor medio de respuesta, quizá se deba a preguntas que no se entendieron correctamente y el valor fue alto, o bien, que se entendieron y la puntuación tendió a ser más elevada que la puntuación real, contrastándose con estudios donde se indica que los individuos que no son conscientes, tienden sistemáticamente a pensar que saben más de lo que realmente es, y por ello, a responder de una manera no real (Gándara, 2012).

Respecto al diagrama de cajas y bigotes se muestra principalmente dónde se ubican el $50 \%$ de las respuestas de los estudiantes para los respectivos ítems. Podemos observar, que para los ítems 7, 9,11 y 13 de la clase Mindfulness, los datos se encuentran muy concentrados entre las respuestas 3 y 4 , cosa que no ocurre en la clase Control. Por otro lado, preguntas con mayor dificultad de compresión para los alumnos, como ocurrió en el caso del ítem 1, la clase control concentra los valores de respuestas en puntuaciones muy elevadas, mientras que en la clase mindfulness se encuentran más esparcidos. En cuanto a la clase Control, podemos observar que para los ítems 4 (referido al caminar sin ser consciente de mi alrededor) y 12 (preocupación por el futuro), el $50 \%$ se encuentran espaciados en un amplio rango de respuesta, mostrando una disconformidad de respuesta por parte de los alumnos. De igual modo ocurre con el ítem 14 en la clase Mindfulness. 


\subsection{Estudio analítico del cuestionario EAP para la Clase Mindfulness y la Clase Control}

\subsubsection{Análisis Global del Cuestionario EAP}

Los resultados mostrados en el análisis de los datos para el cuestionario EAP, muestran que no ha habido diferencias significativas entre ambas clases en el resultado promedio total/alumno mostrándose unos valores promedio totales de respuesta muy parejos entre ambas clases, a diferencia que ocurría en el apartado anterior con el cuestionario MAAS-A, que fue ligeramente superior el Control a la clase Mindfulness. De igual modo, no ha habido una variación significativa de respuesta entre sesiones, tanto para la Clase Control, como para la Clase Mindfulness. Los resultados indican, que los alumnos han ido contestando de manera repetitiva y constante los cuestionarios, obteniéndose puntuaciones promedio muy parejas para cada sesión y entre ambas clases.

En cuanto al análisis de la Parte I y la Parte II, quedan recogidos en el siguiente gráfico:

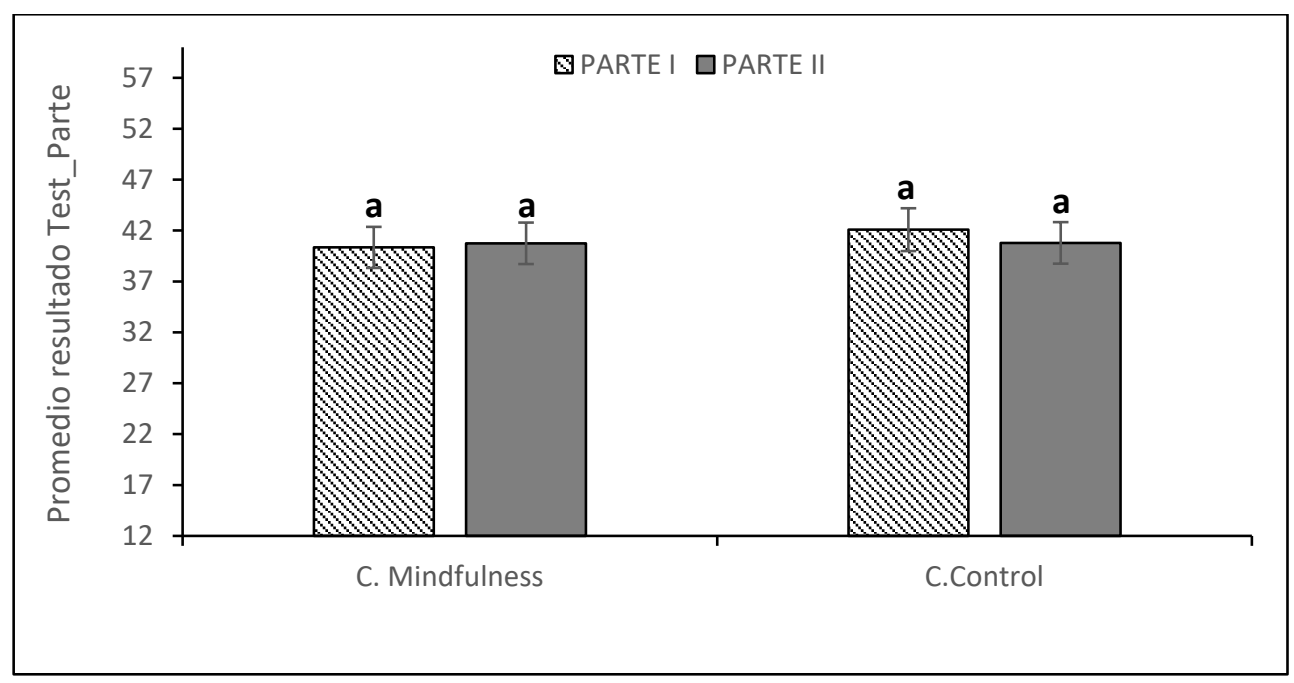

Figura 6. Evolución del promedio respuestas del Test EAP de la Parte I y Parte Il con el tipo clase de estudio.

Se observa que no ha habido variación en el promedio respuesta entre la Parte I y la Parte II, tanto para la clase Mindfulness como para la clase Control, indicando, por tanto, que no se ha experimentado aparentemente un efecto en ningún tipo de mejoría en la puntuación con la aplicación de ejercicios de atención plena en la clase Mindfulness. En cuanto al valor de la puntuación, son puntuaciones de media a elevada, indicando un nivel medio-alto en Atención Plena en ambas clases.

En cuanto a la variable "Momento del día" y la variable "Día", se ha observado que no existen diferencias significativas en ambos casos (Véase Tabla 2).

Tabla 2.

Cuadro resumen entre las variables Momento del día y Día, para la clase Control como para la clase Mindfulness para el cuestionario EAP.

\begin{tabular}{lccccc}
\hline \multicolumn{1}{c}{ Tipo de Clase } & Momento del día & Promedio & $\begin{array}{c}\text { Desviación } \\
\text { Estándar }\end{array}$ & Razón-F & Valor-P \\
\hline C. Mindfulness & Final de la mañana & 41 & 6,39 & 0,19 & 0,6679 \\
C. Mindfulness & Primera hora & 40 & 7,95 & & \\
C. Control & Final de la mañana & 43 & 3,64 & 0,7 & 0,5029 \\
C. Control & Media mañana & 40 & 7,63 & & \\
C. Control & Primera hora & 43 & 8,59 &
\end{tabular}




\begin{tabular}{lccccc}
\hline \multicolumn{1}{c}{ Tipo de Clase } & Momento del día & Promedio & $\begin{array}{c}\text { Desviación } \\
\text { Estándar }\end{array}$ & Razón-F & Valor-P \\
\hline C. Mindfulness & Lunes & 40 & 6,45 & 0,22 & 0,8022 \\
C. Mindfulness & Martes & 40 & 7,95 & & \\
C. Mindfulness & Viernes & 41 & 6,43 & & 0,5029 \\
C. Control & Jueves & 40 & 7,63 & 0,7 & \\
C. Control & Lunes & 43 & 3,64 & & \\
C. Control & Martes & 43 & 8,59 & & \\
\hline
\end{tabular}

Fuente: elaboración propia

\subsubsection{Análisis del Cuestionario EAP estudiado por Ítem}

En el presente punto, se analizó el promedio respuesta de cada ítem dentro de la calificación de la escala del cuestionario EAP por alumno para ambas clases del estudio.

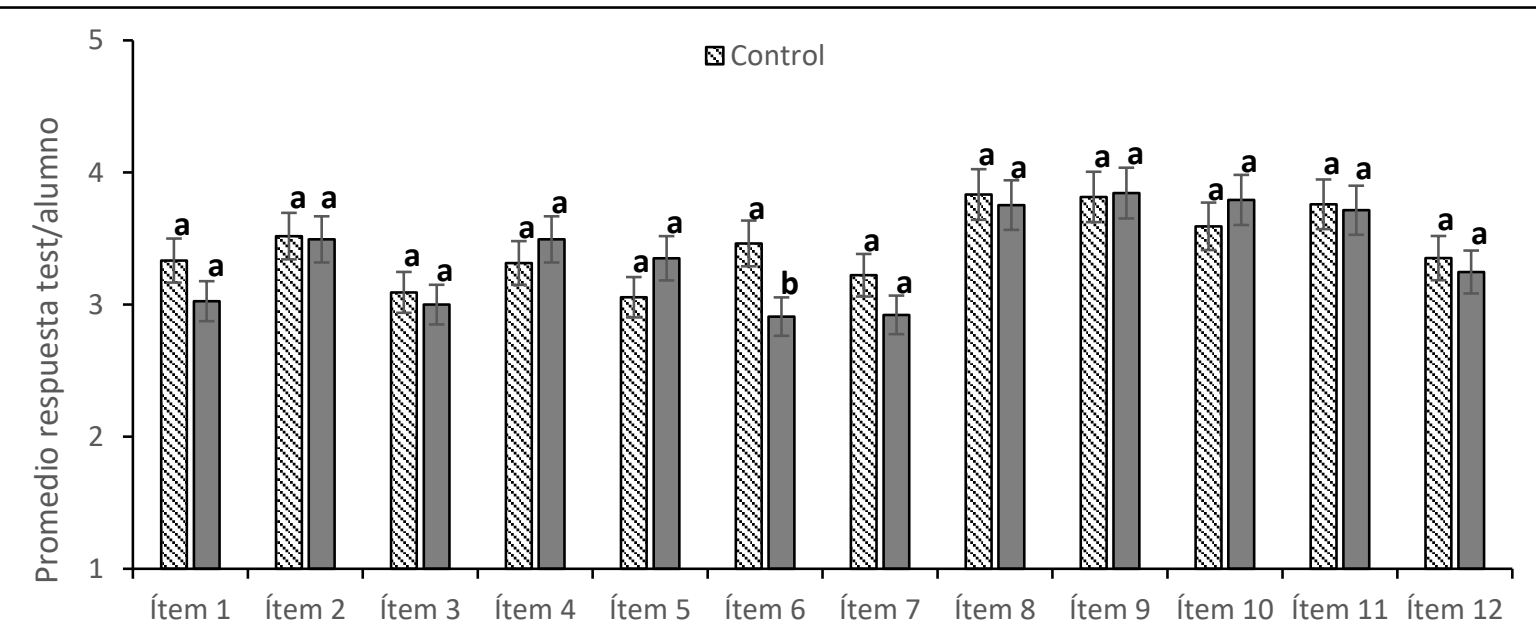

Figura 8. Comparación por Ítem y clase de estudio de los Ítems comprendidos en el cuestionario EAP.

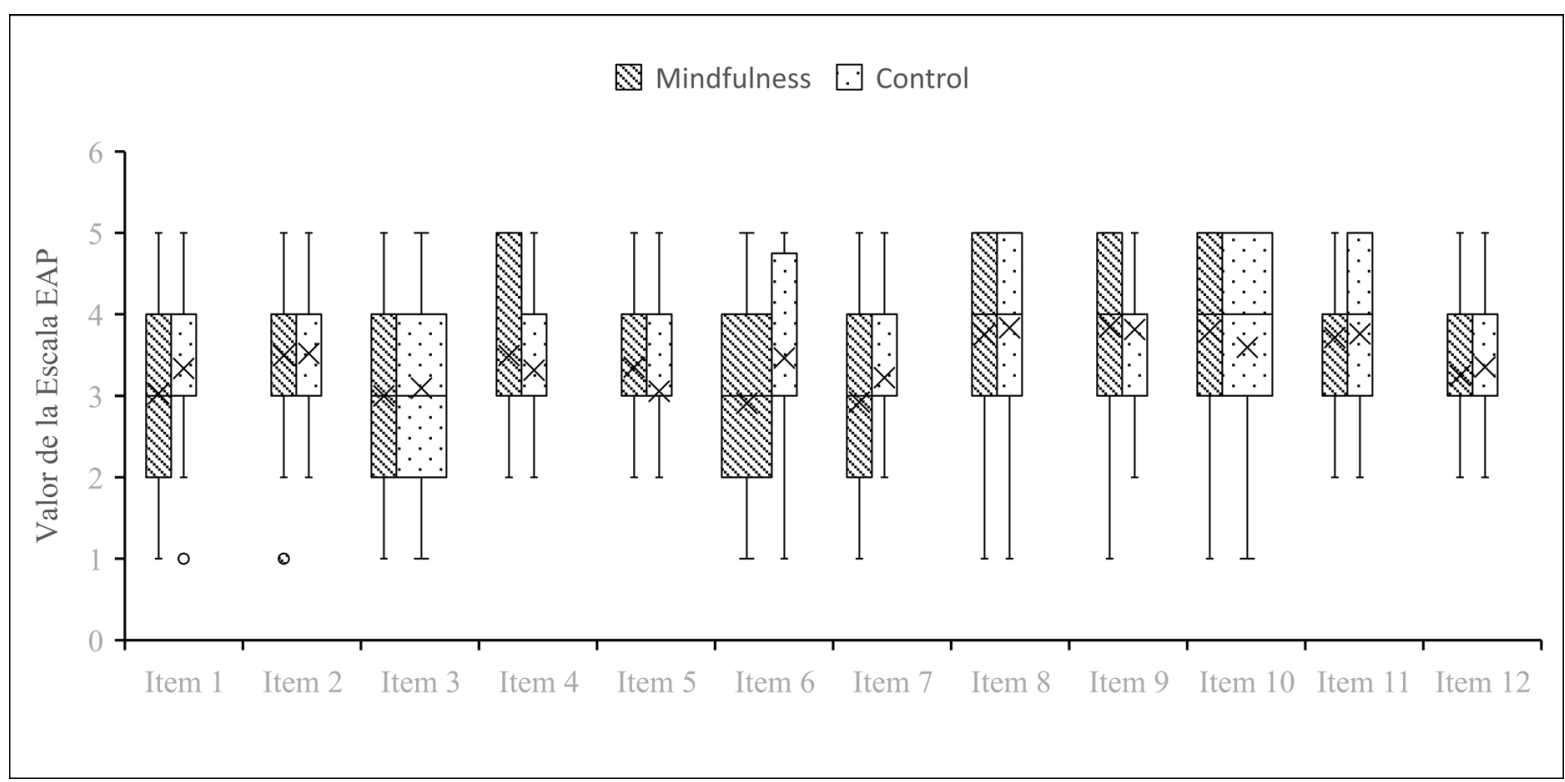

Figura 9. Comparación del rango de respuesta por alumno y clase para los respectivos Ítems del cuestionario EAP. 
Se observa que el promedio respuesta de los distintos ítems pertenecientes a la escala EAP por alumno es muy parejo en la mayoría de las cuestiones, y a diferencia de lo ocurrido en el cuestionario MAAS-A, no se produce una ligera diferencia con tendencia superior en el promedio resultado en la clase Control, sino que el promedio respuesta por alumno de los respectivos ítems son más parejos entre la clase Control y la clase Mindfulness. En cuanto a diferencias observadas en las respuestas, únicamente se encontró diferencias significativas en la respuesta del ítem 6 (Cuando voy de casa al instituto me fijo en el camino que recorro), donde se puede observar que la respuesta en el caso de la clase Mindfulness, presenta un valor promedio inferior respecto a la clase Control. De igual modo, se repite la misma tendencia para los ítems 1 (Cuando camino me doy cuenta de los movimientos que hace mi cuerpo) y 7 (Cuando voy al instituto a casa me doy cuenta de los ruidos que hay a mi alrededor), pero sin presentar diferencias significativas, y viéndose de nuevo que la clase donde se practicó ejercicios en meditación mostró un valor promedio respuesta inferior a la clase Control. En el resto de los ítems, los valores entre ambas clases son muy similares, correspondiéndose todos ellos a respuestas de valor alto, indicando una alta capacidad Mindfulness. No obstante, y basándonos en las observaciones en clase donde se vio la dificultad de comprensión del cuestionario EAP, invita a pensar que se trata de respuestas con tendencia al alza por una insuficiente comprensión de la pregunta, y es por ello esa mayor homogenización de respuestas entre ambas clases.

En el Diagrama de Cajas y Bigotes se observa que para los ítems 2 (Cuando me tumbo en un sillón percibo que mis músculos se relajan), 5 (Cuando paseo por el campo me paso el tiempo contemplando y escuchando la naturaleza"), 11 ("A menudo soy consciente de como mi respiración se acelera) y 12 (Soy capaz de recordarme a mí mismo cuando siento, pienso o actúo) de la clase Mindfulness, el $50 \%$ de los datos se concentran entre el valor 3-4, indicando recorrido muy estrecho del rango interquartil, de igual modo ocurre para los ítems 2, 4 (Cuando me cepillo los dientes presto atención a lo que hago, y siento), 5, 7, 9 (Me doy cuenta rápidamente cuando algo me produce alegría) y 12 de la clase Control. En dichos casos, pueden estar indicando que se ha tenido una decisión firme y unísona en el valor respuesta, que viene precedida por una forma de responder mecánica y automática, o bien por una comprensión de la respuesta.

En cuanto a los ítems 1, 3 (Cuando me visto por la mañana me doy cuenta de los movimientos), 4, 6, 7, 8 (Cuando me ducho, siento el agua caer por mi cuerpo), 9 y 10 (Cuando me doy cuenta de las cosas bellas de la vida me siento bien), presentan un rango interquartil muy amplio de respuesta para la clase Mindfulness, indicando una heterogeneidad en la respuesta por parte del alumno. De igual modo, para los ítems 3, 6, 8, 10 y 11 presentan un rango interquartil muy amplio de respuesta para la clase Control.

\subsection{Estudio analítico del cuestionario CAMM para la Clase Mindfulness y la Clase Control}

\subsubsection{Análisis Global del Cuestionario CAMM estudiado}

Los resultados mostrados en el análisis de los datos para el cuestionario CAMM, muestran que no ha habido diferencias significativas entre ambas clases en el resultado promedio total/alumno, mostrándose unos valores promedio totales de respuesta muy parejos entre ambas clases. De igual modo, no ha habido diferencias significativas entre la puntuación promedio y el número de sesiones para la clase Mindfulness. Por otro lado, sí se puede observar que hubo diferencias significativas en la puntuación promedio para la sesión 4 de la clase Control, siendo superior su puntuación respecto al resto de sesiones.

Respecto a las diferencias entre las partes y clase, de igual modo, no se ha visto una diferencia significativa entre la Parte I y la Parte II, tal y como se muestra a continuación: 


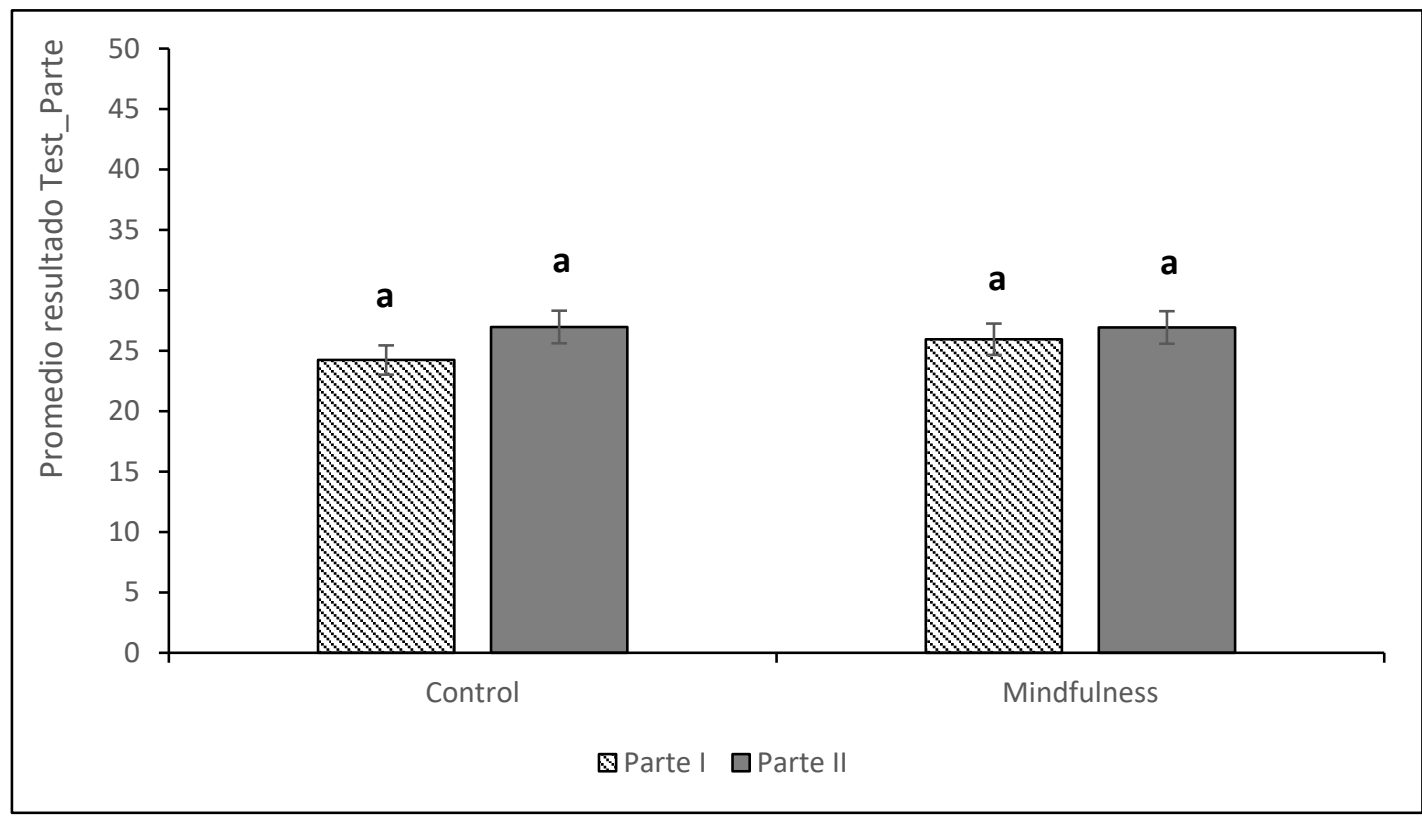

Figura 10. Evolución del promedio respuestas del Test CAMM de la Parte I y Parte II con el tipo clase de estudio.

En la clase Control, se observa un ligero aumento de la puntuación promedio en la segunda parte del estudio. No obstante, dicho aumento no resulta ser significativo en comparación con la Parte I. De igual modo, ocurre lo mismo en la clase Mindfulness, donde se observa un aumento de la puntuación promedio, pero mucho menos marcado que en el caso control, y no siendo de igual manera significativo. Por lo general, se observa que la puntuación promedio presenta una variación muy reducida entre la Parte I y la Parte II para ambas partes, invitando a pensar que ambas clases se han comportado de una manera muy parecida compartiendo una comprensión del cuestionario similar. Como en los casos anteriores, y tal como indicó León (2008), existe un problema del sesgo de la deseabilidad social y la tendencia a contestar siempre igual, explicando quizá el comportamiento de los datos y su poca variación de la puntuación promedio entre ambas partes y para ambas clases.

En cuanto al valor de la puntuación, se observa que son puntuaciones medias-altas, debido a que tal y como se ha indicado anteriormente, las puntuaciones bajas en el cuestionario CAMM se corresponden con un alto grado de atención plena, mientras que puntuaciones elevadas se correlacionan negativamente con dicha variable (Timoneda, 2017).

En cuanto a la variable "Momento del día" y variable "Día" se ha observado que no existen diferencias significativas en el momento del día en ambas clases. (Véase Tabla 3).

Tabla 3.

Cuadro resumen entre las variables Momento del día y Día, para la clase Control como para la clase Mindfulness para el cuestionario CAMM.

\begin{tabular}{lccccc}
\hline \multicolumn{1}{c}{ Tipo de Clase } & Momento del día & Promedio & $\begin{array}{c}\text { Desviación } \\
\text { Estándar }\end{array}$ & Razón-F & Valor-P \\
\hline C. Mindfulness & Final de la mañana & 27 & 5,82 & 0,52 & 0,4742 \\
C. Mindfulness & Primera hora & 26 & 4,51 & & \\
C. Control & Final de la mañana & 24 & 5,38 & 1,19 & 0,3106 \\
C. Control & Media mañana & 24 & 4,97 & &
\end{tabular}




\begin{tabular}{|c|c|c|c|c|c|}
\hline Tipo de Clase & Momento del día & Promedio & $\begin{array}{c}\text { Desviación } \\
\text { Estándar }\end{array}$ & Razón-F & Valor-P \\
\hline C. Control & Primera hora & 27 & 6,26 & & \\
\hline C. Mindfulness & Lunes & 27 & 5,88 & 0,3 & 0,7432 \\
\hline C. Mindfulness & Martes & 26 & 4,51 & & \\
\hline C. Mindfulness & Viernes & 27 & 5,92 & & \\
\hline C. Control & Jueves & 24 & 4,97 & & \\
\hline C. Control & Lunes & 24 & 5,38 & 1,2 & 0,32 \\
\hline C. Control & Martes & 27 & 6,26 & & \\
\hline
\end{tabular}

Fuente: elaboración propia

\subsubsection{Análisis del Cuestionario CAMM estudiado por Ítem}

En la figura 17 y 18 observamos que el promedio respuesta de los distintos ítems pertenecientes a la escala del cuestionario CAMM por alumno es en la mayoría de los casos resultados muy parejo por ítem y clase. A nivel global, se observa que las respuestas por parte de los alumnos pertenecientes a la clase Mindfulness, son ligeramente más elevadas en puntuación en la escala que en la clase Control, correspondiéndose con un nivel inferior en atención plena que respecto a la clase Control. Resultados que se contrastan de igual modo con resultados obtenidos por otros autores (Bruin et al., 2011), donde la clase Control obtiene resultados en atención plena superiores a la clase Mindfulness.

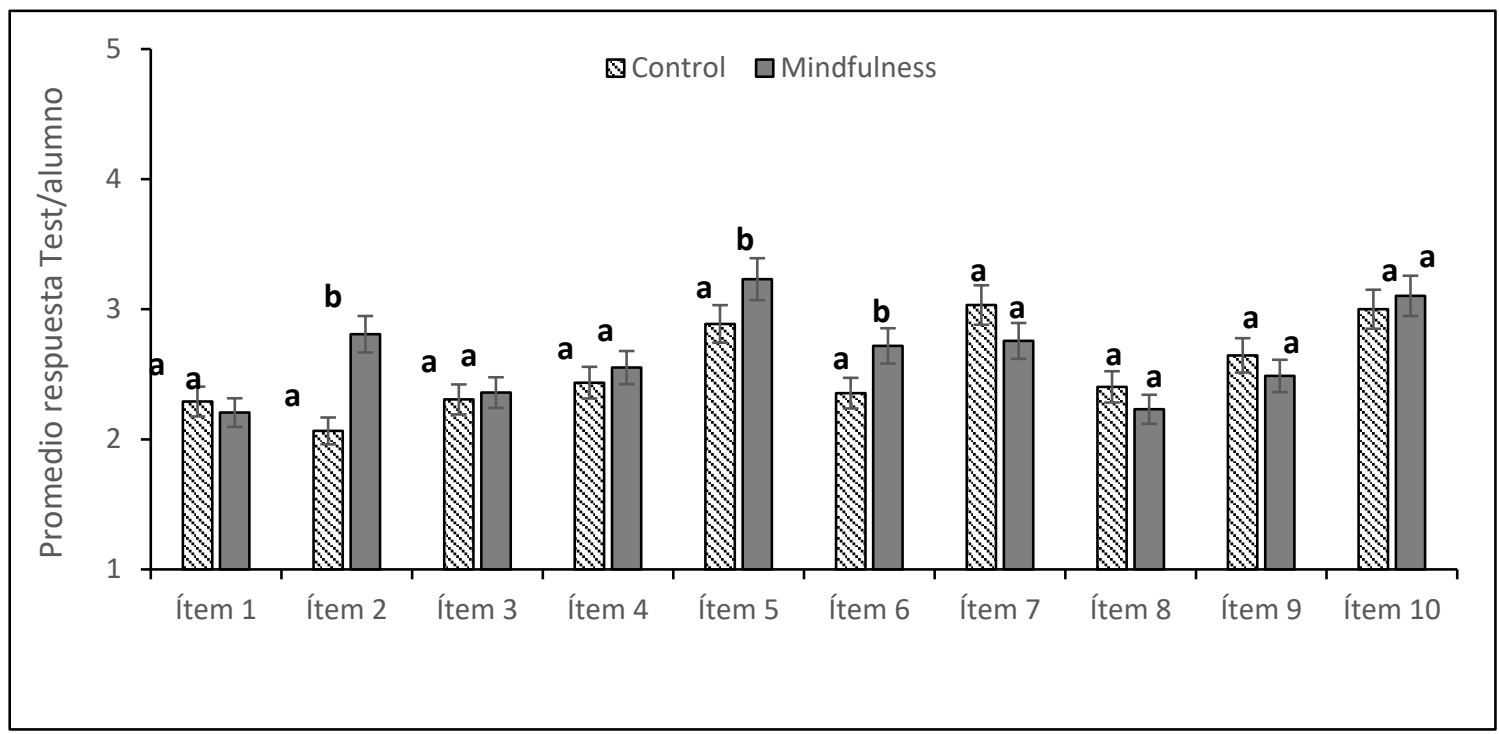

Figura 12. Comparación por Ítem y clase de estudio de los Ítems comprendidos en el cuestionario CAMM. 


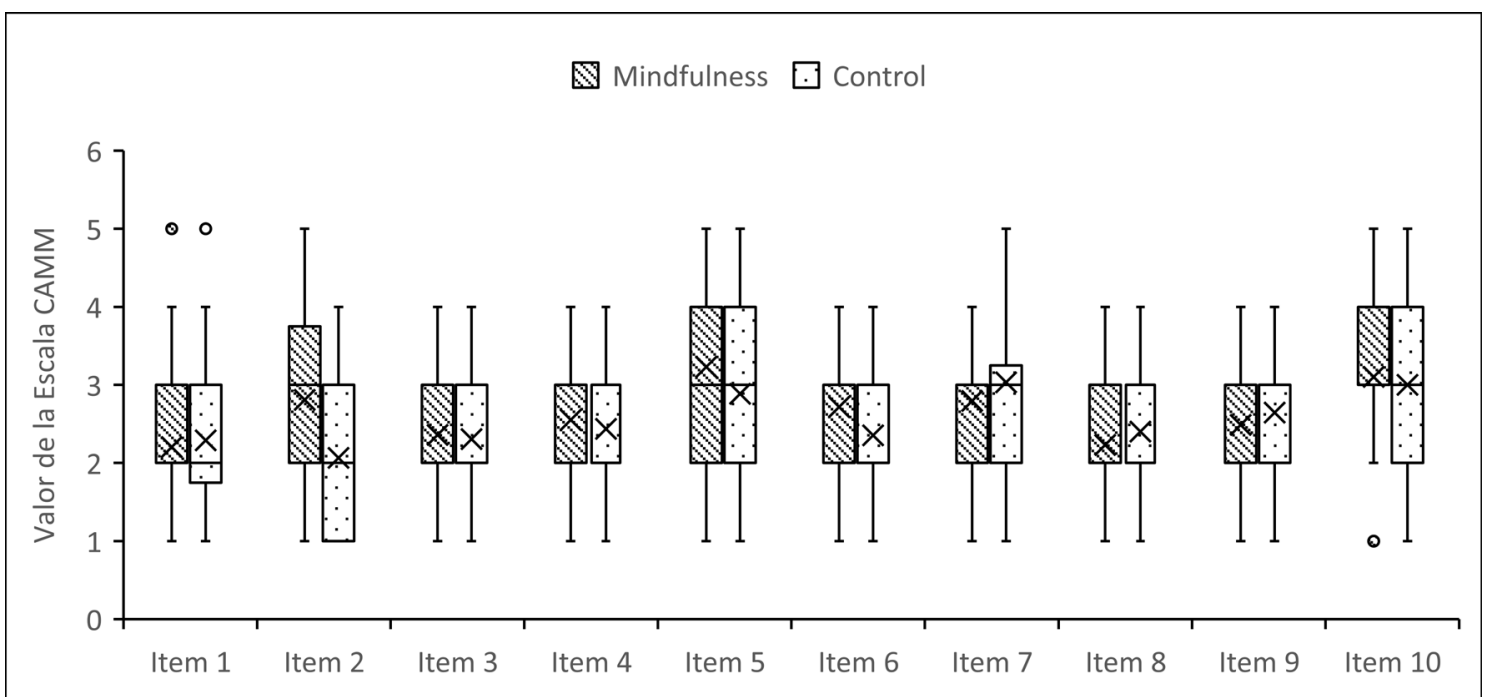

Figura 13. Comparación del rango de respuesta por alumno y clase para los respectivos ítems del cuestionario CAMM.

En cuanto a las diferencias significativas halladas en los diferentes ítems incluidos en el cuestionario CAMM, se observa que existen diferencias estadísticamente significativas para los ítems 2 (En la escuela o instituto, camino de clase a clase sin darme cuenta de lo que estoy haciendo), 5 (Aparto de mi mente los pensamientos que no me gustan) y 6 (Me es difícil prestar atención a una sola cosa en cada momento) entre ambas clases, mostrando valores superiores en el promedio respuesta /alumno en la clase Mindfulness que respecto a la clase Control.

En el Diagrama de Cajas y Bigotes podemos observar, que, a diferencia del resto de cuestionarios analizados, el cuestionario CAMM ha sido el único donde el $50 \%$ de los valores para ambas clases se concentran en un rango intercuartil muy reducido, indicando muy poca variabilidad en la selección de respuesta por parte de los alumnos. No obstante, encontramos en los ítems 2, 5 y 10 (Soy capaz de parar los sentimientos que no me gustan) un rango intercuartil amplio, indicando una variación elevada de respuesta por parte de los alumnos para dichas cuestiones. Para los mismos ítems, esa variación de respuesta se produce al unísono entre ambas clases, mostrándose de manera inversa en el orden de respuesta en el ítem 2, la misma variación de respuesta en el ítem 5 y una variación de respuesta amplía únicamente para la clase Control en el ítem 10.

\subsection{Comparativa del resultado global de los cuestionarios MAAS-A, EAP y CAMM}

En la figura 14 se ha expresado el promedio respuesta total de todos los alumnos, juntando tanto la clase Control y la clase Mindfulness, en tanto por uno para cada tipo de cuestionario analizado en el presente proyecto: 


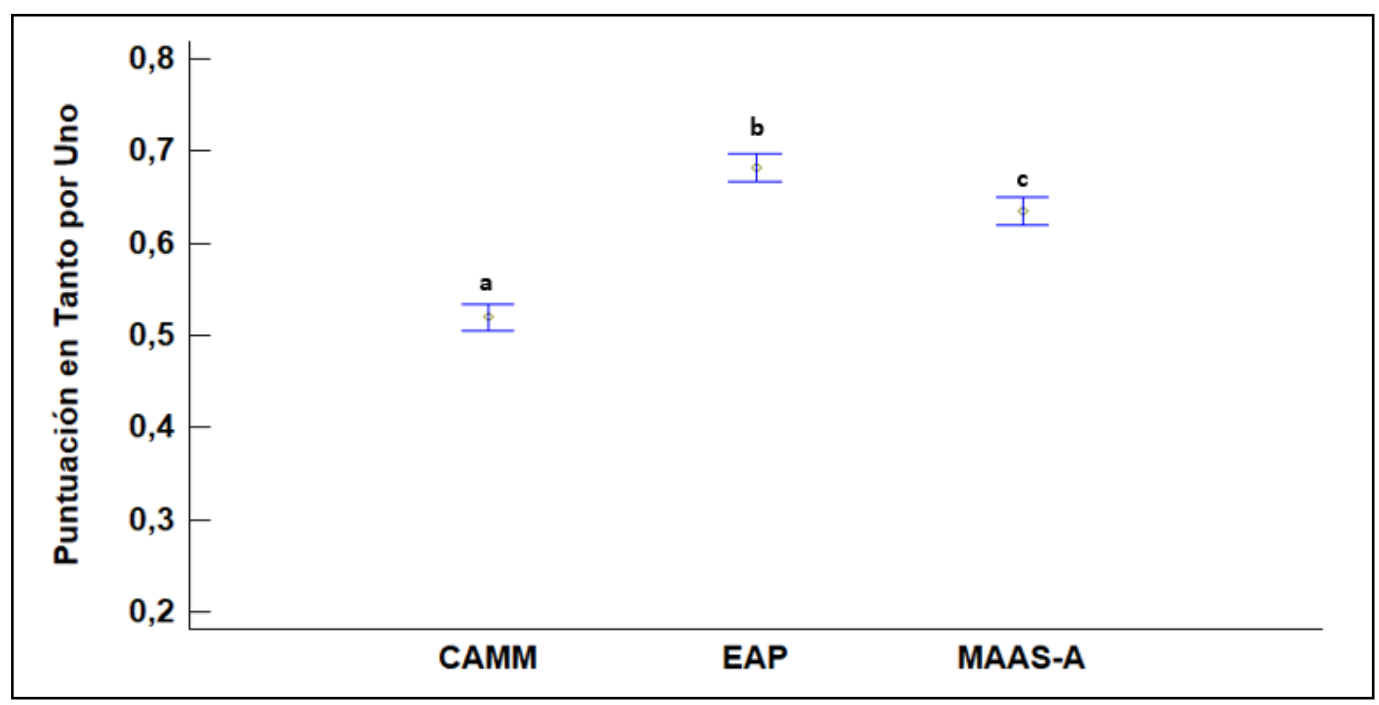

Figura 14. Expresión en tanto por uno de los cuestionarios CAMM, EAP y MAAS-A analizados en el presente proyecto.

Se puede observar la expresión en tanto por uno de los resultados generales de cada alumno de ambas clases para cada cuestionario. Podemos comprobar que existen diferencias significativas entre ellos, correspondiéndose los valores más bajos de las puntuaciones en tanto por uno al cuestionario CAMM, lo cual se corresponde con un alto nivel en atención plena. Por otro lado, podemos observar, que para los cuestionarios EAP y MAAS-A, donde puntuaciones elevadas se correlacionan de manera positiva con un alto nivel en mindfulness, muestran que en el caso del cuestionario EAP, las puntuaciones globales de los estudiantes de ambas clases expresadas en tanto por uno, son significativamente superiores al cuestionario MAAS-A. Se indica así una tendencia al alza a la hora de puntuar para este tipo de cuestionario. Por otro lado, el cuestionario MAAS-A, es posiblemente la prueba que mejor se entendió y más se correlacionó con el grado en Mindfulness de los alumnos para ciertos ítems del cuestionario, ya que, a nivel global, de igual modo que en el resto, se tendió a valores al alza obteniendo puntuaciones elevadas.

\section{Conclusiones}

A la vista de los análisis estadísticos realizados, podemos determinar que ambas clases han obtenido calificaciones altas en los tres cuestionarios realizados, sin mostrar diferencias estadísticas entre ellas. A la vista está, que la evolución del promedio respuesta entre ambas poblaciones a lo largo de las sesiones del proyecto ha sido constante, invitando a pensar una tendencia a contestar siempre igual por parte de los alumnos, lo cual se contrastaría con otros estudios llevados a cabo por León (2008).

La clase denominada Mindfulness, la cual ha recibido entrenamiento por vía de audios en meditación, se ha visto que, en todos los cuestionarios realizados, su promedio respuesta, así como su calificación, ha sido igual o inferior a la clase Control. Este resultado coincide con lo mencionado por Bruin et al. (2011), donde adolescentes sin experiencia en meditación obtuvieron calificaciones más altas que aquellos con experiencia.

En cuanto a la variabilidad de respuesta en la escala para cada ítem de los distintos cuestionarios mostrada por los diagramas de Cajas y Bigotes, podemos observar que el cuestionario MAAS-A es el que mayor variabilidad de respuesta de las opciones de la escala para cada ítem ha obtenido por parte de los alumnos de ambas clases, indicando una posible mayor comprensión 
en las preguntas de dicho cuestionario. En cuanto al cuestionario EAP, se ha visto que la variabilidad de respuesta para la clase Mindfulness sigue siendo muy elevada en comparación con la clase Control, la cual muestra un rango intercuartílico estrecho en la mayoría de sus respuestas, pudiendo indicar que la clase sin experiencia contestase de manera repetitiva mientras que la clase que ejercitaba la meditación lo hiciese tras una lectura más profunda variando en un rango mayor la respuesta. Por último, el cuestionario CAMM es el que menor variabilidad de respuesta presenta en la escala para cada ítem, tanto en la clase Control como en la clase Mindfulness, pudiendo indicar una lectura menos profunda y una tendencia a contestar de manera repetitiva los mismos valores durante todas las sesiones.

Los resultados obtenidos nos permiten concluir que la aplicación de audios durante seis sesiones continuas no ha servido para mostrar diferencias estadísticamente significativas entre las clases, quedando así refutada nuestra hipótesis. No obstante, sí se han visto pequeños matices a la hora de analizar la respuesta de los ítems por separado, indicando una posible mayor comprensión en las preguntas, precedida por una mayor variabilidad en la elección de respuestas, así como una posible mayor sinceridad de respuesta en ciertos ítems por parte de la clase Mindfulness. Este resultado nos lleva a la hipótesis de que el proceso de aplicación de las sesiones de Mindfulness puede haber influido en los resultados. En este sentido, consideramos que los audios seleccionados para el estudio no fueron adecuados para la edad de la muestra de estudio, siendo necesario buscar otros recursos adaptados a adolescentes y/o para personas principiantes. No obstante, en este último caso, nos encontramos con una falta de audios de mindfulness adaptados a estas edades, lo cual nos abre la posibilidad de crear este recurso didáctico para los centros educativos.

Otra hipótesis que se nos plantea a partir de los resultados obtenidos es la posibilidad de que el tipo de asignatura (Módulo de Electricidad y Automatismos Electrónicos) haya influido en los resultados, ya que la materia no está relacionada explícitamente con el mindfulness, lo cual puede llevar a ciertas reticencias por parte del alumnado. En este sentido, sería interesante realizar un estudio comparativo entre materias para ver si las respuestas del alumnado se ven influenciadas según la asignatura donde se realizan los ejercicios. Además, se incluye la posibilidad de que la poca variabilidad entre las respuestas se deba al número elevado de tests realizados por estudiantes, lo cual nos lleva a plantear un estudio paralelo utilizando un número inferior de instrumentos de análisis.

En este sentido, para futuras líneas de investigación sería interesante repetir el mismo estudio, aumentando la duración del entrenamiento en Mindfulness, así como el número de actividades y técnicas, no basándonos únicamente en el audio como medio de entrenamiento.

\section{Referencias bibliográficas}

Almansa, G., Budía, A. M., López, J. L., Márquez, J. M., Martínez, A. I., Palacios, B., Peña, J.M., Santafé, P., Zafra, J., Fernández-Ozcota, E., y Sáenz-López, P. (2014). Efecto de un Programa Mindfulness sobre variables motivacionales y psicológicas en Educación Primaria. E-motion. Revista de Educación, Motricidad e Investigación, 41(3), 120-133.

Anand, U., y Sharma, M. P. (2014). Effectiveness of a mindfulness-based stress reduction program on stress and well-being in adolescents in a school setting. Indian Journal of Positive Psychology, 5(1), 17.

Biegel, G., y Brown, K. W. (2010). Assessing the efficacy of an adapted in class mindfulness-based training program for school-age children: A pilot study. Mindful Schools. Recuperado de https://www.mentalhealthexcellence.org/wp-content/uploads/2013/10/MindfulSchools-Pilot-Study-Whitepaper.pdf 
Broderick, P. C., y Metz, S. (2009). Learning to Breathe: A pilot trial of mindfulness currículum for adolescents. Advances in School Mental Health Promotion, 2(1), 35-46.

Bruin, E. I., Zijlstra, B.J., Weijer-Bergsma, E., y Bögels, S.M. (2011). The Mindful Attention Awareness Scale for Adolescent (MAAS): Psychometric Properties in a Dutch Sample. Faculty of Behavioral and Social Science. Research institute of Child Development and Education. Amsterdam: Springer.

Carroll, J.B. (1993). Cognitive abilities. Reino Unido: Cambridge University Press.

Calvete, E., Sampedro, A., y Orue, I. (2014). Propiedades psicométricas de la versión española de la "escala de atención y consciencia plena para adolescentes (MAAS-A)". Behavioral Psychlogy, 22(2), 277-291.

Franco, C., Mañas, I., Cangas, A. J., y Gallego, J. (2011). Exploring the effects of a mindfulness program for students of secondary school. International Journal of Knowledge Society Research (IJKSR), 2(1), 14-28.

Gándara, J. (2012). El Efecto Dunning-Kruger. Med Psicosom, 102, 7-8.

Greco, L.A. Baer, R.A. y Smith, G.T. (2011). Assessing mindfulness in children and adolescents: development and validation of the Child and Adolescent Mindfulness Measure (CAMM). Psychological assessment, 23(3), 606-614.

León, B. (2008). Atención plena y rendimiento académico en estudiantes de enseñanza secundaria. European Journal of Education and Psychology, 1(3), 17-26.

Lutz, A., Slagter, H., Dunne, J.D., y Davidson, R.J. (2008). Attention regulation and monitering in meditation. Trends in Cognitive Sciencies, 12(4), 163-169.

Napoli, M., Krech, P. R., y Holley, L.C. (2005). Mindfulness training for elementary school students: The attention academy. Journal of Applied School Psychology, 21(1), 99-125.

Rosaen, C., y Benn, R. (2006). The experience of transcendental meditation in middle school students: A qualitative report. Explore: The Journal of Science and Healing, 2, 422-425.

Siegel, D., Germer, C. K., y Olendzki, A. (2009). Mindfulness: What Is It? Where Did It Come From? En F. Didonna (Ed.), Clinical handbook of Mindfulness, 17-35. New York, Springer.

Siegel, R. (2011). La solución mindfulness: Prácticas cotidianas para problemas cotidianos. Bilbao: España.

Simón, V. y Alonso, M. (2013). Meditaciones de Mindfulness y Autocompasión (5rd.Ed) [CDROM]. Ibiza: Magrama Estudios.

Timoneda, A. (2017). Programa de mindfulness en educación primaria para niños comprendidos entre 11 y 12 años. Zaragoza: Universidad de Zaragoza.

Wall, R. B. (2005). Tai chi and mindfulness-based stress reduction in a Boston public middleschool. Journal of Pediatric Health Care, 19(4), 230-237.

Waters, L., Barsky, A., Ridd, A., y Allen, K. (2015). Contemplative education. A systematic, evidence-based review of the effect of mediation interventions in schools. Educational Psychology Review, 27(1), 103-134.

Zoogman, S., Goldberg, S. B., Hoyt, W. T., y Miller, L. (2015). Mindfulness interventions with youth: A meta-analysis. Mindfulness, 6(2), 290-302. https://doi.org/10.1007/s12671-0130260-4 